\title{
Vector boson production in association with jets at CMS
}

\author{
Anastasia Grebenyuk ${ }^{* \dagger}$ \\ IIHE-ULB \\ E-mail: agrebeny@ulb.ac.be
}

The production of vector bosons (W, Z, gamma) in association with jets is a stringent test of perturbative QCD and is a background process in searches for new physics. Total and differential cross-section measurements of vector bosons produced in association with jets in proton-proton collisions performed by the CMS collaboration at the LHC are presented. The measurements are compared to the predictions of event generators and theoretical calculations.

XXVI International Workshop on Deep-Inelastic Scattering and Related Subjects (DIS2018) 16-20 April 2018

Kobe, Japan

\footnotetext{
*Speaker.

${ }^{\dagger}$ on behalf of the CMS collaboration
} 


\section{Introduction}

Measurements of vector boson production in association with jets provide fundamental tests of perturbative quantum chromodynamics. A precise knowledge of the kinematic dependencies in processes with large jet multiplicity is essential to exploit the discovery potential of the LHC experiments. Comparison of the measurements with predictions allows further Monte Carlo (MC) generator development and improvement of our understanding of the prediction uncertainties. Furthermore, the production of a massive vector boson together with jets is an important background to a number of standard model processes.

\section{Z+jets at $13 \mathrm{TeV}$}

We present a cross section measurement for $\mathrm{Z}$ production in association with $N$ or more jets ( $p p \rightarrow Z / \gamma *+\geq N$ jets) at a centre-of-mass energy of $13 \mathrm{TeV}$, combining the electronic and muonic decay channels of the $\mathrm{Z}$ boson [1]. The measurement is performed with data from the CMS detector [2] recorded in 2015 at the LHC corresponding to $2.19 \mathrm{fb}^{-1}$ integrated luminosity.

The measured differential cross sections are compared to four calculations. The first one is MADGRAPH5_aMC@NLO (denoted MG5_aMC) interfaced with PYTHIA8 for parton showers and hadronisation. MG5_aMC includes matrix elements (MEs) computed at leading order (LO) for the five processes $p p \rightarrow Z+N$ jets, $N=0 \ldots 4$ and matched to the parton shower using the $k_{T}$-MLM scheme. The second one is MG5_aMC interfaced with PyThIA8, with MEs computed at NLO the three processes $p p \rightarrow Z+N$ jets, $N=0 \ldots 2$ and merged with the parton shower using the FxFx scheme. The PyTHIA8 tune CUETP8M1 is used for both MG5_aMC samples.

The third calculation uses GENEVA1.0-RC2 MC program (GE), where an NNLO calculation for Drell-Yan (DY) production is combined with higher-order resummation. Logarithms of the 0 -jettiness resolution variable, $\tau$, also known as beam thrust, are resummed at NNLL' (denoted NNLL $_{\tau}{ }^{\text {). }}$. The Pythia8 tune CUETP8M1 is used.

The fourth calculation performed at NNLO calculation for $Z+1$ jet using the $N$-jettiness subtraction scheme $\left(\mathrm{N}_{\text {jetti }}\right)$. The nonperturbative correction is applied.

The measured cross sections are unfolded to the particle level. The phase space is restricted to events with a $Z$ boson mass between 71 and $111 \mathrm{GeV}$ and both leptons with $p_{\mathrm{T}}>20 \mathrm{GeV}$ and $|\eta|<2$.4. Jets are required to have $p_{\mathrm{T}}>30 \mathrm{GeV},|y|<2.4$ and a spatial separation from the dressed leptons of $R>0.4$.

Fig. 1 (left) shows the measured cross section as a function of the exclusive jet multiplicity. Agreement between the measurement and the MG5_aMC prediction is observed. The GENEVA prediction describes the measured cross section up to a jet multiplicity of 2, but fails to describe the data for higher jet multiplicities, where one or more jets arise from the parton shower. Fig. 1 (middle) shows measured cross section as a function of the transverse momentum of the $Z$ boson for events with at least one jet. The best model for describing the measurement at low $p_{\mathrm{T}}$, below the peak, is NLO MG5_aMC. The high- $p_{\mathrm{T}}$ region, where GENEVA and NLO MG5_aMC are expected to have similar accuracy (NLO), is equally well described by the two. Fig. 1 (right) shows the jet transverse momenta for the $1^{\text {st }}$ leading jet. The LO MG5_aMC predicted spectrum differs from the measurement, showing a steeper slope in the low $p_{\mathrm{T}}$ region. The comparison with NLO MG5_aMC and $\mathrm{N}_{\text {jetti }}$ NNLO calculation shows that adding NLO terms cures this discrepancy. The GENEVA prediction shows good agreement with the data. 

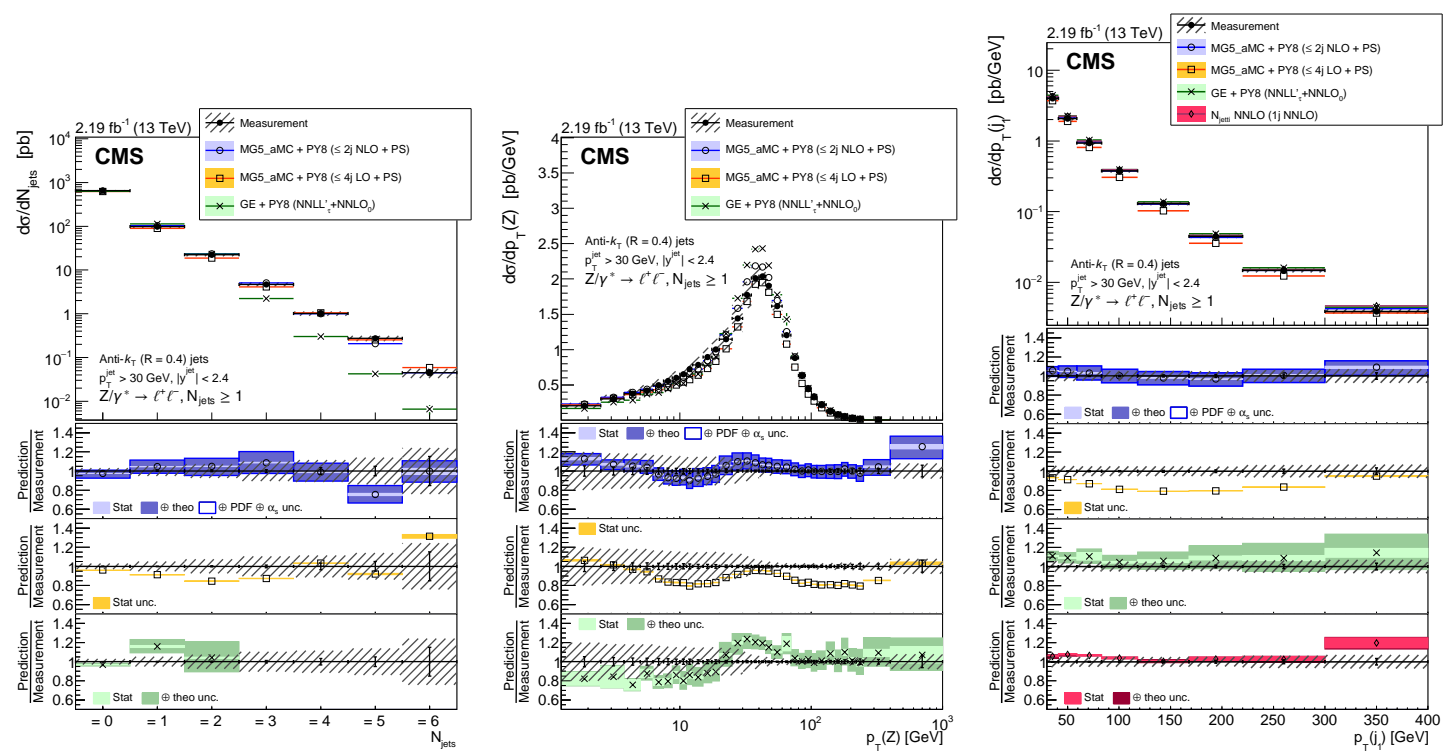

Figure 1: Measured $Z+$ jets cross section as a function of the jet exclusive multiplicity (left), $p_{T}(\mathrm{Z})$ for events with at least one jet (middle) and transverse momentum of the first jet (right) [1].
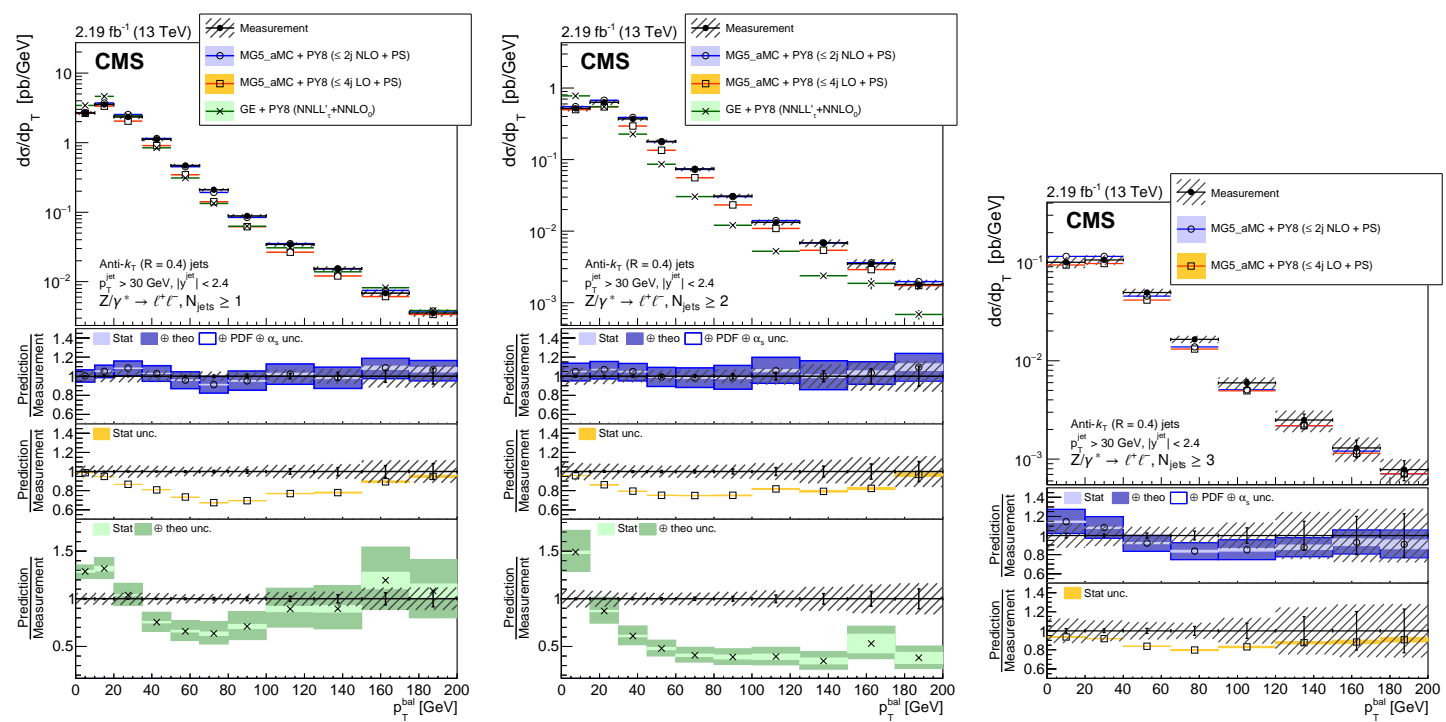

Figure 2: Measured $Z+$ jets cross section as a function of the $p_{\mathrm{T}}$ balance between the $Z$ boson and the accompanying jets for events with at least one (left), two (middle) and three (right) jets [1].

The balance in transverse momentum between the jets and the $Z$ boson, $p_{\mathrm{T}}^{\text {bal }}$, is shown in Figs. 2 for inclusive jet multiplicities of 1,2 , and 3 . The measurement is in good agreement with NLO MG5_aMC predictions. The NLO correction is important for the description of hadronic activity beyond the jet acceptance used in this analysis. An imbalance in the event, i.e. $p_{\mathrm{T}}^{\text {bal }}$ not equal to zero, requires two partons in the final state with one of the two out of the acceptance, Figs. 2 (left). Such events are described with NLO accuracy for the NLO MG5_aMC sample and LO accuracy for the two other samples. 


\section{W+jets at $13 \mathrm{TeV}$}

The differential measurement of the $\mathrm{W}(\mu v)+$ jets production in the muon decay channel at a center-of-mass energy of $13 \mathrm{TeV}$ is presented [3]. The same data as in the $\mathrm{Z}+\mathrm{jets}$ analysis collected by the CMS detector are analysed. The measured $\mathrm{W}+$ jets differential cross sections are compared to two multileg ME calculations, the NLO prediction and LO predictions, as desribed in the previous section. The measured differential cross sections are also compared to the fixed-order calculation based on the $\mathrm{N}$-jettiness subtraction scheme $\left(\mathrm{N}_{\mathrm{jetti}}\right)$ at $\mathrm{NNLO}$ for $\mathrm{W}+1$-jet production. The nonperturbative correction is applied.

The measured cross sections are unfolded to the particle level. The phase space is restricted to events with a muon with $p_{\mathrm{T}}>25 \mathrm{GeV}$ and $|\eta|<2.4$ and which are further required to be in the transverse mass peak region for $\mathrm{W}$ bosons, defined by $m_{\mathrm{T}}>50 \mathrm{GeV}$. Jets are required to have $p_{\mathrm{T}}>30 \mathrm{GeV},|y|<2.4$ and a spatial separation from the dressed muons of $R>0.4$.

Figure 3 shows the measured cross section as a function of the exclusive jet multiplicity (left), jet transverse momenta for the $1^{\text {st }}$ leading jet (middle) and the scalar sum of jet transverse momenta $\left(H_{\mathrm{T}}\right)$ for inclusive jet multiplicities of 1 . The LO MG5_aMC prediction underestimates data in contrast to NLO MG5_aMC FxFx and NNLO at low and moderate $p_{\mathrm{T}}$ of leading jet and $H_{\mathrm{T}}$.
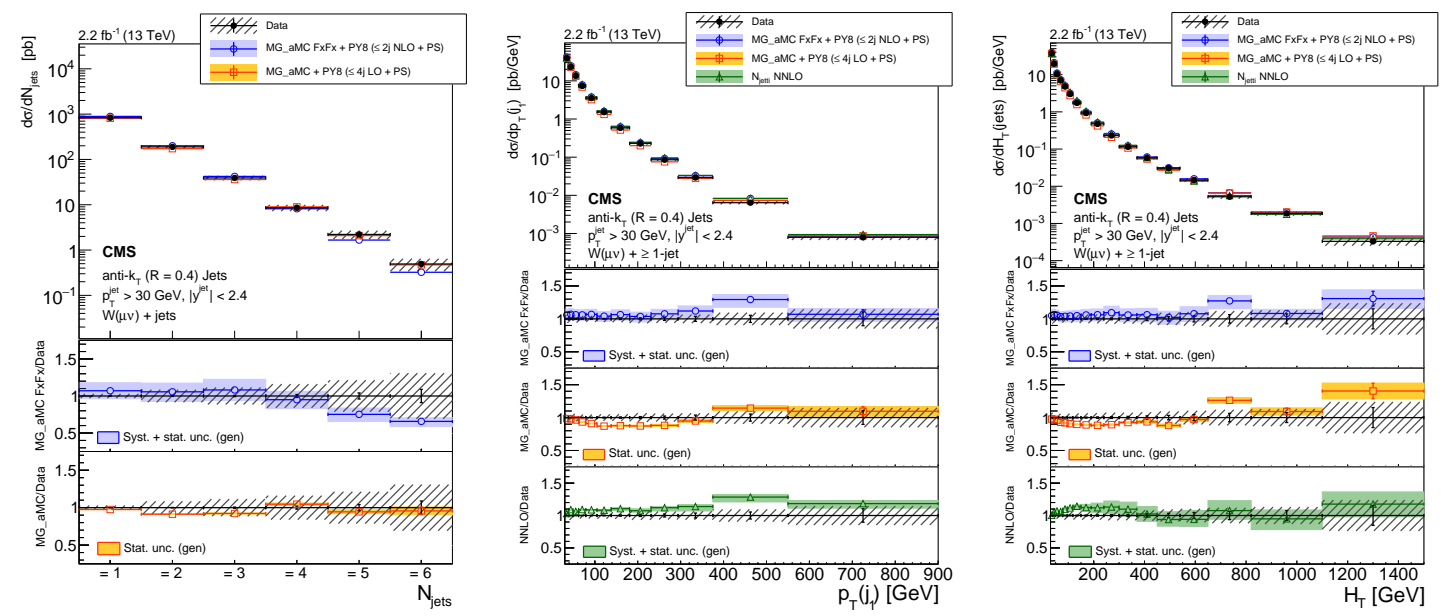

Figure 3: Measured $W+$ jets cross section as a function of the jet exclusive multiplicity (left), leading jet $p_{\mathrm{T}}$ (middle) and the $H_{\mathrm{T}}$ observable for events with at least one jet (right) [3].

Figure 4 shows the differential cross sections as functions of angular variables: the azimuthal separation $\Delta \varphi\left(\mu, j_{1}\right)$ between the muon and the jet, and the angular distance between the muon and the closest jet $\Delta R(\mu$, closest jet $)$ in events with one or more jets. $\Delta \varphi$ is sensitive to the implementation of particle emissions and other nonperturbative effects modeled by parton showering algorithms in $\mathrm{MC}$ generators. The $\Delta R$ observable probes the angular correlation between the muon emitted in the $\mathrm{W}$ boson decay and the direction of the closest jet. In the collinear region (small $\Delta R$ values), it is sensitive to the modeling of $\mathrm{W}$ boson radiative emission from initial- or final-state quarks. For the analysis of the $\Delta R$ distribution, jets in the event are required to have $p_{\mathrm{T}}>100$ $\mathrm{GeV}$, with the leading jet $p_{\mathrm{T}}>300 \mathrm{GeV}$. This selection results in a boosted topology, where two jets recoil against each other and one of them can lose a significant amount of energy to the decay 

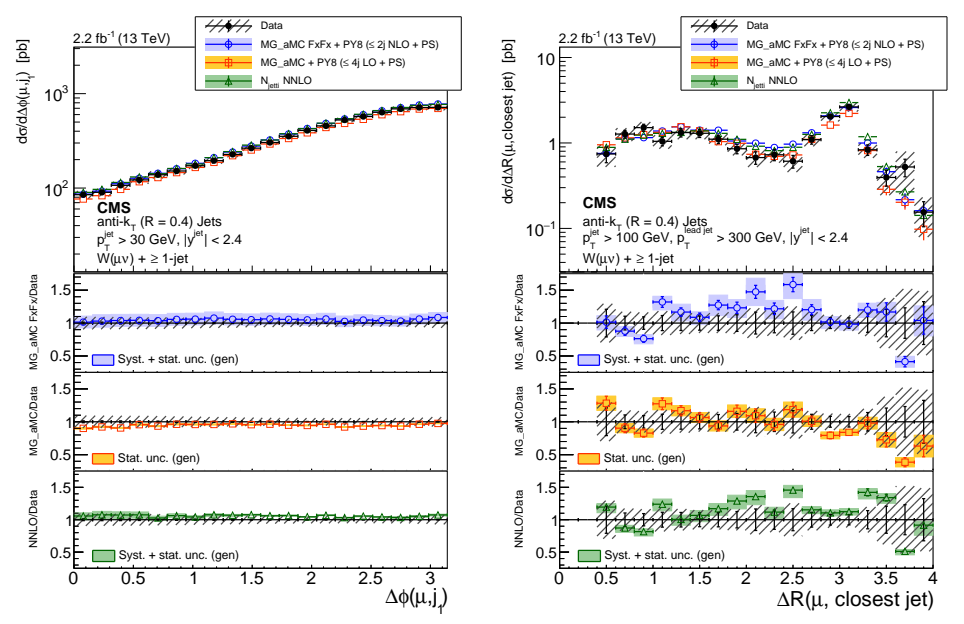

Figure 4: Measured $W+$ jets cross section as a function of angular variables $\Delta \varphi\left(\mu, j_{1}\right)$ (left) and $\Delta R(\mu$, closest jet) (right) for events with at least one jet [3].

products of the emitted real W boson. Both $\Delta \varphi\left(\mu, j_{1}\right)$ and $\Delta R(\mu$, closest jet $)$ are described by the predictions within the uncertainties, but there are some differences.

\section{EWK Z+2jets at $13 \mathrm{TeV}$}

A measurement of the electroweak (EW) production of two jets in association with a $\mathrm{Z}$ boson in proton-proton collisions at $13 \mathrm{TeV}$ is presented, based on data recorded in 2016 by the CMS experiment corresponding to an integrated luminosity of $35.9 \mathrm{fb}^{-1}$ [4].

The pure electroweak production of the $l l \mathrm{jj}$ final state $(l$ combines electrons and muons and the jets $\mathrm{j}$ corresponding to the quarks produced in the hard interaction) includes production via the vector boson fusion process, with its distinctive signature of two jets with both large energy and separation in pseudorapidity. The signal is defined in the kinematic region with dilepton invariant mass $m_{l l}>50 \mathrm{GeV}$, jet transverse momentum $p_{\mathrm{Tj}}>25 \mathrm{GeV}$, and the invariant mass of the two tagging jets $m_{\mathrm{jj}}>120 \mathrm{GeV}$.

A multivariate analysis is used to separate signal events from the large DY+jets background. The main discriminating variables are the dijet invariant mass, $m_{\mathrm{jj}}$, and the pseudorapidity separation, $\Delta \eta_{\mathrm{jj}}$. The $m_{j j}$ and $\Delta \eta_{\mathrm{jj}}$ distributions for data and simulated samples for muon channel are shown in Fig. 5 (left and middle). Signal events are simulated at leading order with MG5_aMC, interfaced with PYTHIA8. Background DY events are simulated with NLO MG5_aMC+PYTHIA8. Good agreement between the data and the MC expectations is observed. Six different variables are used in the multivariate analysis. The output of the discriminator is built by training a boosted decision tree (BDT) to achieve an optimal separation between the EW Zjj and DY Zjj processes independently in the dielectron and dimuon channels. Fig. 5 (right) shows the distribution for transformed BDT discriminants, BDT' $=\tanh ^{-1}((\mathrm{BDT}+1) / 2)$. The signal strength, defined for the $l l \mathrm{jj}$ final state is extracted from the fit to the BDT output distribution. The signal cross section is measured to be $\sigma(\mathrm{EW} l \mathrm{ljj})=534 \pm 20$ (stat) \pm 57 (syst) $\mathrm{fb}=534 \pm 60$ (total) fb and is in agreement with the SM LO prediction. 

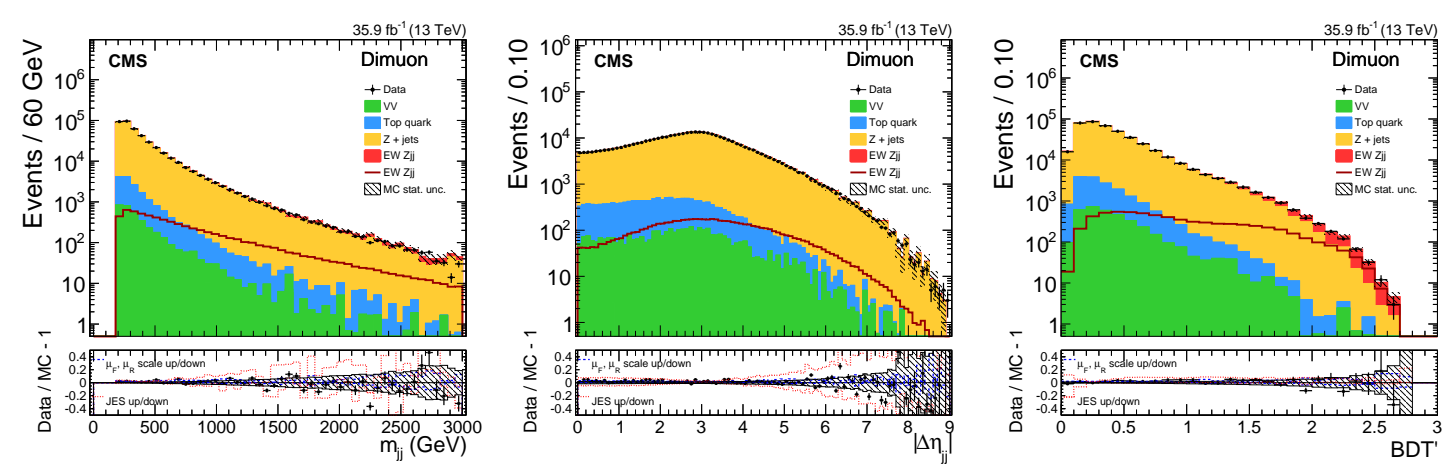

Figure 5: Data and simulated $m_{\mathrm{jj}}$ (left) and $\Delta \eta_{\mathrm{jj}}$ (midle) for the dimuon event selection together with the distribution for transformed BDT discriminants (right) [4].

In the paper the efficiency of the third jet veto, which is not applied in the analysis, is also studied. Gap activity fraction (or gap veto efficiency), defined as fraction of events which do not have reconstructed kinematics above a given threshold of the third jet, is shown in Fig. 6. The events with BDT $>0.92$ are selected which allow to select a signal-enriched region with a similar fraction of signal and background event. Data are compared to MC expectations with only DY

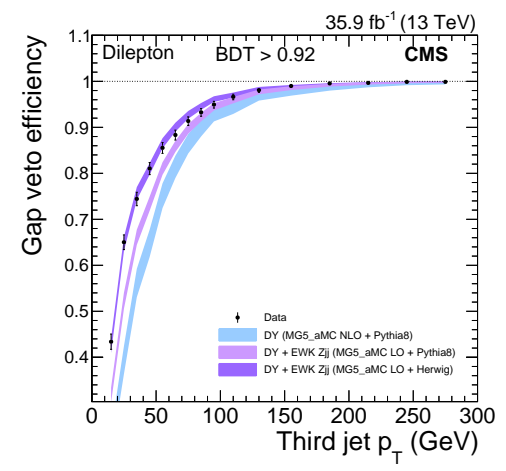

Figure 6: Efficiency of a gap activity veto as a function of the additional jet $p_{\mathrm{T}}$ [4].

events and with DY+signal events generated with the PYTHIA8 or the HERWIG++ parton shower models. Data disfavour backgorund only predictions and are in reasonable agreement with the presence of the signal for both parton shower predictions.

\section{References}

[1] CMS Collaboration, "Measurement of differential cross sections for $\mathrm{Z}$ boson production in association with jets in proton-proton collisions at $\sqrt{s}=13 \mathrm{TeV}$ ", arXiv:1804.05252.

[2] CMS Collaboration, "The CMS experiment at the CERN LHC", JINST 3 S08004 (2008).

[3] CMS Collaboration, "Measurement of the differential cross sections for the associated production of a $W$ boson and jets in proton-proton collisions at $\sqrt{s}=13$ TeV", Phys. Rev. D 96 (2017) 072005.

[4] CMS Collaboration, "Electroweak production of two jets in association with a $\mathrm{Z}$ boson in proton-proton collisions at $\sqrt{s}=13$ TeV", Submitted to: Eur. Phys. J. C, arXiv:1712.09814. 